Trends in Horticulture (2018) Volume 1 doi: $10.24294 /$ th.v1i3.549

\title{
Arbuscular mycorrhizal symbiosis improves growth and antioxidative response of Stevia rebaudiana (Bert.) under salt stress.
}

Siamak Shirani Bidabadi ${ }^{*}$, Majid Masoumian²

${ }^{1}$ Isfahan University of Technology, Isfahan, Iran, siamakshirani.upm@gmail.com

${ }^{2}$ Department of Agriculture, Iranian Research Organization for Science and Technology

\section{ABSTRACT}

To investigate the possible role of arbuscular mycrrhizal fungi (AMF) in alleviating the negative effects of salinity on Stevia rebaudiana (Bert.), the regenerated plantlets in tissue culture was transferred to pots in greenhouse and inoculated with Glomus intraradices. Salinity caused a significant decrease in chlorophyll content, photosynthesis efficiency and enhanced the electrolyte leakage. The use of AMF in salt -affected plants resulted in improved all above mentioned characteristics. Hydrogen peroxide and malondialdehyde (MDA) contents increased in salt stressed plants while a reduction was observed due to AMF inoculation. CAT activity showed a significant increase up to $2 \mathrm{~g} / \mathrm{l}$ and then followed by decline at $5 \mathrm{~g} / \mathrm{l} \mathrm{NaCl}$ in both $\mathrm{AMF}$ and non-AMF treated stevia, however, AMF inoculated plants maintained lower CAT activity at all salinity levels ( 2 and $5 \mathrm{~g} / \mathrm{l})$. Enhanced POX activities in salt- treated stevia plants were decreased by inoculation of plants with AMF. The addition of $\mathrm{NaCl}$ to stevia plants also resulted in an enhanced activity of SOD whilst, AMF plants maintained higher SOD activity at all salinity levels than those of non-AMF inoculated plants. AMF inoculation was capable of alleviating the damage caused by salinity on stevia plants by reducing oxidative stress and improving photosynthesis efficiency.

Keywords: Arbuscular mycorrhizal fungi; salinity; Stevia rebaudiana; antioxidant enzymes; oxidative stress; photosynthesis

\section{Introduction}

Stevia (Stevia rebaudiana Bertoni) is a perennial shrub widely planted in many countries of the world with great potential as a natural sweetener source, up to 150 times sweeter than sugar, but with no calories. Therefore, it has been applied as a substitute for saccharose, and in the treatment of diabetes mellitus, obesity, and hypertension and in caries prevention ${ }^{[20,22]}$. According to reports by Hajar et al. (2014) stevia has successfully been able to adapt to different growing areas in terms of climate and soils ${ }^{[12]}$. However, for an economical production, crop irrigation is required ${ }^{[21]}$. Although stevia has shown different degrees of sensitivity or resistance against salinity, there is often a tendency for a relation between growth and salinity according to reports of the scientific literatures where in, the higher salinity level the less growth of stevia ${ }^{[6,14,25]}$.

Because, around $40 \%$ of the arable lands in the world have insufficient rainfall to support economically viable agriculture, the influence of saline water irrigation on stevia growth has attracted more attention ${ }^{[28]}$. Plants grown in fields are surrounded by various microorganisms like bacteria and fungi that help and improve the plant growth under various stress conditions ${ }^{[7]}$. The application of Arbuscular mycorrhizal fungi (AMF) as a biologically based strategy could be an efficient alternative to mitigate the harmful effects of plant crops exposed to salt. AMFs alleviate salt stress by several possible mechanisms, including maintaining higher activities of antioxidant enzymes, improving nutrient uptake and providing a higher accumulation of osmosolutes ${ }^{[9,23]}$. Under salinity stress conditions, AMFs improve the uptake mechanism in plants by supplying the essential nutrients; thereby the plant recovers the water balance machinery, resulting in the enhancement of its tolerance against salt stress ${ }^{[1,3]}$. Some experiments have been conducted to assess the response of some plants such as Panicum turgidum ${ }^{[13]}$, Ephedra aphylla Forssk ${ }^{[1]}$, 
sweet basil ${ }^{[8]}$, apple seedlings ${ }^{[32]}$ and Populus catayana ${ }^{[31]}$ to salinity and possible role of AMF in enhancing the salt tolerance. In Citrus plants, leaf area, photosynthesis and phosphorus uptake were enhanced by AMF colonization of plants $^{[27]}$. According to reports of Reis et al. (2015), stevia is suitable to be grown in semiarid regions, if well irrigated, even with a relatively high salinity, but only one harvest is possible ${ }^{[22]}$. Therefore, it was hypothesized that, AM inoculation could be capable of alleviating the damage and restrictions caused by salinity stress on stevia plants by improving photosynthesis efficiency and reducing oxidative stress.

\section{Materials and method}

The plantlets were obtained according to Cantabella et al. (2017) ${ }^{[4]}$ from micro-propagated stevia shoot cultures grown on Murashige and Skoog (MS) medium, supplemented with 3\% (w/v) sucrose, $0.6 \%(\mathrm{w} / \mathrm{v})$ agar and $0.4 \%(\mathrm{w} / \mathrm{v})$ activated charcoal. For elongation and rooting, shoots with three internodes were transferred to half strength MS medium without growth regulator. Under these conditions, the shoots elongated and rooted in 7 weeks. All cultures were maintained at $25 \pm 2{ }^{\circ} \mathrm{C}$ in a growth chamber under the controlled photoperiod of $16 \mathrm{~h}$ light and $8 \mathrm{~h}$ dark cycle with a light intensity of 3000- 4000 lux provided by white fluorescent lamps. The rooted shoots were taken out of the medium and washed with distilled water to remove medium attached to the roots. The plantlets were then acclimatized in pots containing a mixture of perlite and peat (1:2) in a controlled growth chamber under the same controlled photoperiod and temperature condition as mentioned above, for the nodal segment culture stage. After 8 weeks of acclimatization to in vivo conditions, the S. rebaudiana plants were transplanted to pots $(2 \mathrm{~L})$ containing the same proportion of substrate used for acclimatization. Seven days later, the plant tips were cut in order to obtain the uniform size of all plants.

\subsection{Plant inoculation}

AMF treatments were inoculated with $250 \mathrm{~g}$ (per pot) soil inoculated with Glomus intraradices, by placing a thin layer of maycorrhizal inoculums around the roots of plantlets below the surface of the soil in pots directly prior to the growing of the stevia plants to promote fungal inoculation of plant roots. Counterparts of non-AM treatment were received volumetric sterilized soils free of spores.

\subsection{Salinity treatment}

The modified Hoagland's solution according to Zeng et al. (2013) ${ }^{[33]}$, supplemented with different concentrations of $\mathrm{NaCl}$ to get concentrations of 0,2 and $5 \mathrm{~g} / 1$ were used for irrigation in normal pots as well as in pots with mycorrhizal inocolum. The rate of irrigation was $250 \mathrm{ml}$ for each treatment twice a week with these solutions at the beginning of and throughout the experiment period. At the end of the experimental period ( 8 weeks), the plants were harvested for growth, physiological and biochemical estimation.

\section{Chlorophyll determination, chlorophyll fluorescence and electrolyte leakage}

Chlorophyll fluorescence was measured using a fluorometer (Walz, Effeltrich, Germany). The photochemical efficiency of PSII was calculated as the ratio Fv/Fm for each segment. The relative chlorophyll (Chl) content was measured with a portable leaf chlorophyll meter (SPAD 502, Minolta Co., Osaka, Japan). Electrolyte leakage (EL) was measured by using a conductivity meter according to Ozden et al. $(2009)^{[18]}$. The samples were divided into equal sized pieces $(0.3 \mathrm{~g}$ per treatment) and placed in culture vessels containing $15 \mathrm{ml}$ of distilled water and were left for $24 \mathrm{~h}$ at room temperature. The initial conductance of the solution was measured using a conductivity meter. The tubes were then autoclaved at $115^{\circ} \mathrm{C}$ for $10 \mathrm{~min}$ and final reading was taken following autoclaving and an additional $24 \mathrm{~h}$ incubation at room temperature. EL (\%) was calculated using the following formula:

$$
\mathrm{EL}(\%)=\frac{\text { The initial conductance of the solution }}{\text { The final conductance of the solution }} \times 100
$$

\subsection{Oxidative stress parameters}

Lipid peroxidation: (MDA) and $\mathrm{H}_{2} \mathrm{O}_{2}$ measurement 
The concentration of malondialdehyde (MDA) which is a product of lipid peroxidation was assessed by the thiobarbituric acid (TBA) according to Wang et al. (2009) ${ }^{[30]}$, as $1 \mathrm{~g}$ fresh leaves were detached from glasshouse grown seedlings, placed in a mortar containing $5 \mathrm{ml} 0.6 \%$ TBA in $10 \%$ trichloroacetic acid (TCA) and ground with a pestle. The mixture was heated at $100{ }^{\circ} \mathrm{C}$ for $15 \mathrm{~min}$. These samples were cooled on ice for $5 \mathrm{~min}$, then, the mixtures were centrifuged at 5,000 rpm for $10 \mathrm{~min}$. The absorbances of the supernatant at 450, 532 and $600 \mathrm{~nm}$ wavelengths were recorded and MDA content was calculated on a fresh weight basis using the following formula:

$$
\left(\mathrm{nmol} \mathrm{MDA} \mathrm{g}^{-1} \mathrm{FW}\right)=6.45\left(\mathrm{OD}_{532}-\mathrm{OD}_{600}\right)-0.56\left(\mathrm{OD}_{450}\right) \times 1000 \text {. }
$$

Hydrogen peroxide $\left(\mathrm{H}_{2} \mathrm{O}_{2}\right)$ was assessed spectrophotometrically after the reaction with potassium iodide (KI), according to the method presented in Velikova and Loreto (2005) ${ }^{[29]}$. Leaf tissues $(1 \mathrm{~g})$ were ground and homogenized in a mortar containing $10 \mathrm{ml} 0.1 \%$ trichloroacetic acid (TCA). The homogenate was centrifuged at 12,000 rpm for 15 min. Afterwards, $0.5 \mathrm{ml}$ of the supernatant was added to $0.5 \mathrm{ml}$ of $10 \mathrm{mM}$ potassium phosphate buffer (pH 7.0$)$ and $1 \mathrm{ml}$ reagent (1 M KI in fresh double distilled water) and then absorbance of the supernatant was read at $390 \mathrm{~nm}$. The blank probe was prepared using $0.1 \%$ TCA in the absence of leaf extract. The content of $\mathrm{H}_{2} \mathrm{O}_{2}$ was calculated applying a standard curve prepared by identified concentrations of hydrogen peroxide.

\subsection{Antioxidative metabolism}

\subsubsection{Enzyme extraction and analysis}

Frozen-leaf samples $(0.2 \mathrm{~g})$ were ground in liquid nitrogen and stored at $-80{ }^{\circ} \mathrm{C}$ until assay. The enzyme extract for superoxide dismutase (SOD), proxidase (POX) and catalase (CAT) was prepared by mixing frozen samples with $2 \mathrm{ml}$ extraction buffer containing $0.1 \mathrm{M}$ potassium phosphate buffer, $\mathrm{pH} 7.5$ and $0.5 \mathrm{mM}$ ethylenediamintetra acetic acid (EDTA). The extract was centrifuged for $20 \mathrm{~min}$ at $12,0009 \mathrm{~g}$ and $4{ }^{\circ} \mathrm{C}$. Then the supernatant was used for enzymatic assay. Assay of SOD activity (expressed as unit per milligram of protein) was based on reduction of nitroblue tetrazolium (NBT) according to the method used by Padmaja et al. (2011) ${ }^{[19]}$. A complete reaction mixture contained 1 $\mathrm{ml}$ of the $125 \mathrm{mM}$ sodium carbonate, $0.4 \mathrm{ml}$ of $25 \mathrm{lM} \mathrm{NBT}$ and $0.2 \mathrm{ml}$ of $0.1 \mathrm{mM}$ EDTA added to $0.5 \mathrm{ml}$ of plant extract. The reaction was initiated by adding $0.4 \mathrm{ml}$ of $1 \mathrm{mM}$ hydroxylamine hydrochloride and the absorbance was read at $560 \mathrm{~nm}$ using a spectrophotometer at $5 \mathrm{~min}$ intervals. Units of SOD were expressed as the amount of enzyme required for inhibiting the reduction of NBT by $50 \%$. Catalase activity was measured by the titrimetric method applied by Padmaja et al. (2011) ${ }^{[19]}$. The reaction mixture comprised of $5 \mathrm{ml}$ of $300 \mathrm{lM}$ phosphate buffer (pH 6.8) containing $100 \mathrm{lM}$ hydrogen peroxide $\left(\mathrm{H}_{2} \mathrm{O}_{2}\right)$ and $1 \mathrm{ml}$ of plant extract was prepared and left at $25{ }^{\circ} \mathrm{C}$ for $1 \mathrm{~min}$. The reaction was stopped by adding $10 \mathrm{ml}$ of $2 \%$ sulfuric acid and residual $\mathrm{H} 2 \mathrm{O} 2$ as titrated with potassium permanganate $(0.01 \mathrm{~N})$ till pink color was obtained. Enzyme activity was measured by calculating the decomposition of $1 \mathrm{M} \mathrm{H}_{2} \mathrm{O}_{2}$ per min per mg protein. Assay of peroxidase was also achieved according to the method used by Padmaja et al. (2011) ${ }^{[19]}$. $3.5 \mathrm{ml}$ of phosphate buffer ( $\mathrm{pH} 6.5$ ) was taken into a clean, dry cuvette, $0.2 \mathrm{ml}$ of plant extract and $0.1 \mathrm{ml}$ of freshly prepared O-dianisidine solution was added to it at $28-30{ }^{\circ} \mathrm{C}$ and absorbance was recorded at $430 \mathrm{~nm}$. Then $0.2 \mathrm{ml}$ of 0.2 $\mathrm{mM} \mathrm{H} \mathrm{H}_{2}$ was added and mixed and then the absorbance was read at every $30 \mathrm{~s}$ intervals up to 3 min. A graph was plotted with an increase in absorbance against time. The enzyme activity was expressed per unit time per mg of protein.

\subsubsection{Statistical analysis of data}

Experiments were performed using a completely randomized design. All statistical analyses were carried out with SAS and MSTAT-C computer programs. The data were analyzed by one-way analysis of variance (ANOVA). Mean separations were performed by LSD test. Differences at $\mathrm{p} \leq 0.05$ were considered as significant.

\section{Results}

The effect of arbuscular mycorrhizal fungi (AMF) G. intraradices on the chlorophyll content and photosynthesis efficiency of Stevia under different levels of $\mathrm{NaCl}$ induced salt stress is shown in Figures 1. Salinity treatments significantly lowered chlorophyll content and photosynthesis efficiency (Fv/Fm) of non-AMF inoculated plants (Fig. 1) and the rate of decrease was directly proportional to the salt concentrations. Maximum decrease of $19.40 \%$ and $27.40 \%$, in chlorophyll content and photosynthesis efficiency, respectively was observed at $5 \mathrm{~g} / \mathrm{l} \mathrm{NaCl}$. However, AMF 
inoculated plants showed improved efficiency of photosynthesis and chlorophyll content at all salinity levels (2 and 5 $\mathrm{g} / \mathrm{l} \mathrm{NaCl}$ ) as the increase of chlorophyll and photosynthesis efficiency of AMF inoculated stevia at $5 \mathrm{~g} / \mathrm{l} \mathrm{NaCl}$ induced salt stress was observed 56.52 and $47.61 \%$ respectively, more than non-AMF inoculated plants (Figs. 1 a and b). The data presented in Figure 2 revealed that the electrolyte leakage (EL) of stevia plants was significantly increased by increasing salt levels, but the use of AMF alleviated the negative effect of salinity on electrolyte leakage significantly. Inoculation with AMF caused a 41\% reduction of ion leakage in the stevia plants under $5 \mathrm{~g} / 1$ of $\mathrm{NaCl}$ induced salt stress compared with non-AMF inoculated plants (Figure 2). Malondialdehyde (MDA) and $\mathrm{H}_{2} \mathrm{O}_{2}$ contents increased with an increased salt level irrespective of the AMF treatment used (Figures 3). However, these values in AMF-inoculated stevia plants were significantly lower in both the cases (MDA and $\mathrm{H}_{2} \mathrm{O}_{2}$ ) than the corresponding non-AMF inoculated plants, indicating higher lipid peroxidation in the non-AMF plants, thus supporting the role of AMF colonization in preventing oxidative stress. The content of chlorophyll and photosynthesis efficiency showed a significant negative correlation with MDA and $\mathrm{H}_{2} \mathrm{O}_{2}$ accumulation and electrolyte leakage (Table 1). Data related to the activities of CAT, POX and SOD are summarized in Figures 4, 5 and $\mathbf{6}$. The data in these figures indicated that salt stress caused significant changes in the activities of these antioxidant enzymes in stevia plants. Results showed an increase in CAT activity significantly up to $2 \mathrm{~g} / \mathrm{l}$ and then followed by decline at $5 \mathrm{~g} / \mathrm{l} \mathrm{NaCl}$ in both AMF and non-AMF treated stevia, however, AMF inoculated stevia plants maintained lower CAT activity at all salinity levels $(2$ and $5 \mathrm{~g} / \mathrm{l})$ (Figure 4). POX activities were significantly higher in salt- treated stevia plants than in the non-treated plants (Figure 5), and the inoculated of plants with AMF resulted in decreased POX activities. The addition of $\mathrm{NaCl}$ induced salt stress to stevia plants also resulted in an enhanced activity of SOD whilst, AMF stevia plants maintained higher SOD activity at all salinity levels than those of non-AMF inoculated plants (Figure 6).
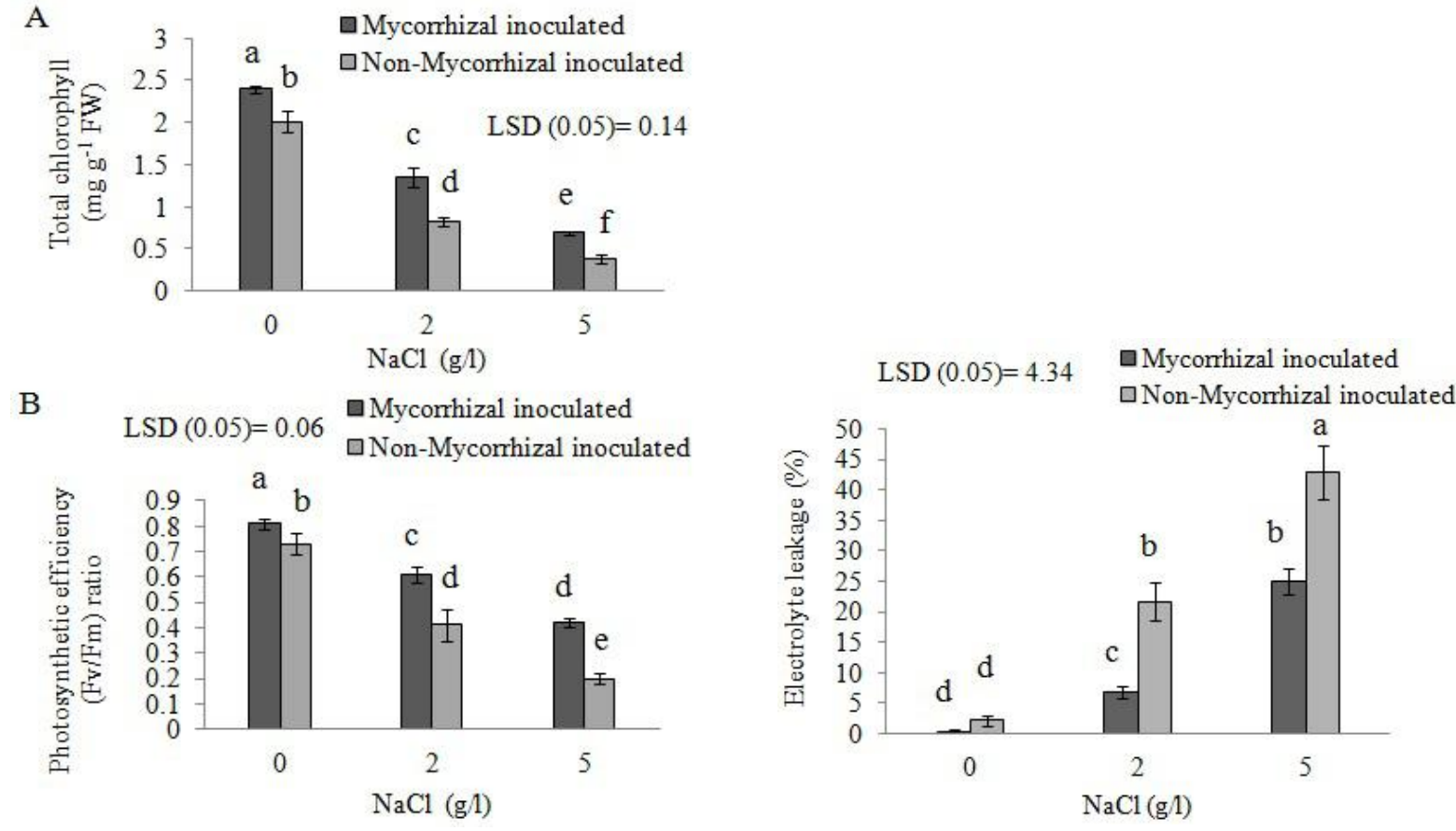

Figure 1; Total chlorophyll and photosynthetic efficiency in Stevia rebaudiana Bertoni plants inoculated with AMF or non-AMF subjected to three different levels of $\mathrm{NaCl}$ induced salt stress. Mean pairs for each treatment followed by different letters are significantly different $(\mathrm{P}<0.05)$ by LSD test; $\mathrm{n}=3$.

Figure 2; Electrolyte leakage in Stevia rebaudiana Bertoni plants inoculated with AMF or non-AMF subjected to three different levels of $\mathrm{NaCl}$ induced salt stress. Mean pairs for each treatments followed by different letters are significantly different $(\mathrm{P}<$ 0.05 ) by LSD test; $\mathrm{n}=3$. 
A

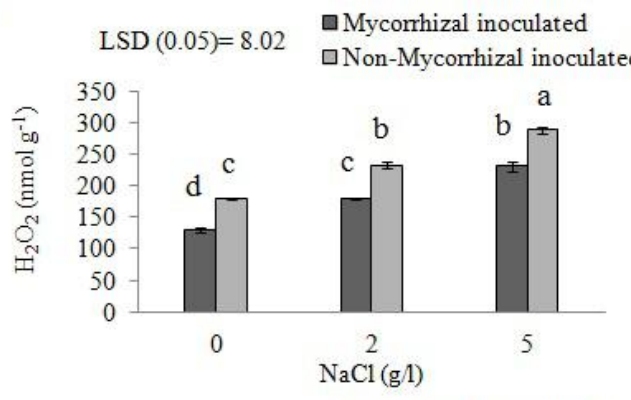

B

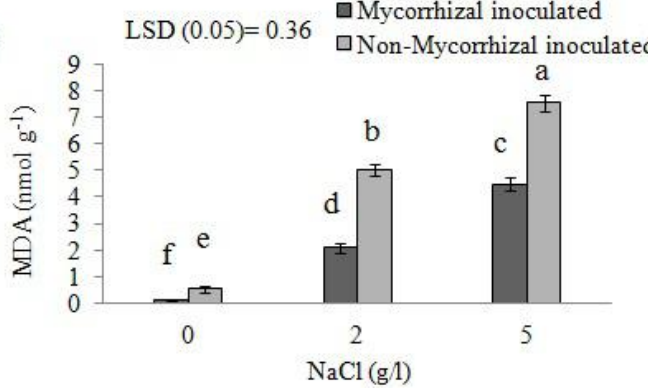

Figure 3; Oxidative stress indicated by $\mathrm{H}_{2} \mathrm{O}_{2}$ and MDA production in Stevia rebaudiana Bertoni plants inoculated with AMF or non-AMF subjected to three different levels of $\mathrm{NaCl}$ induced salt stress. Mean pairs for each treatments followed by different letters are significantly different $(\mathrm{P}<0.05)$ by LSD test; $\mathrm{n}=3$.

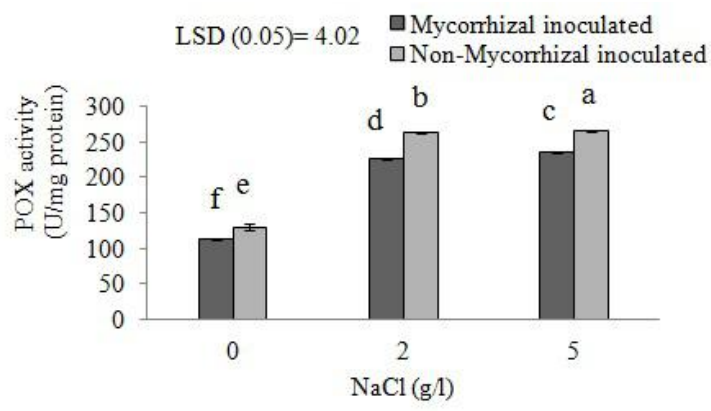

Figure 5; POX activity in Stevia rebaudiana Bertoni plants inoculated with AMF or non-AMF subjected to three different levels of $\mathrm{NaCl}$ induced salt stress. Mean pairs for each treatments followed by different letters are significantly different $(\mathrm{P}<0.05)$ by LSD test; $\mathrm{n}=3$.

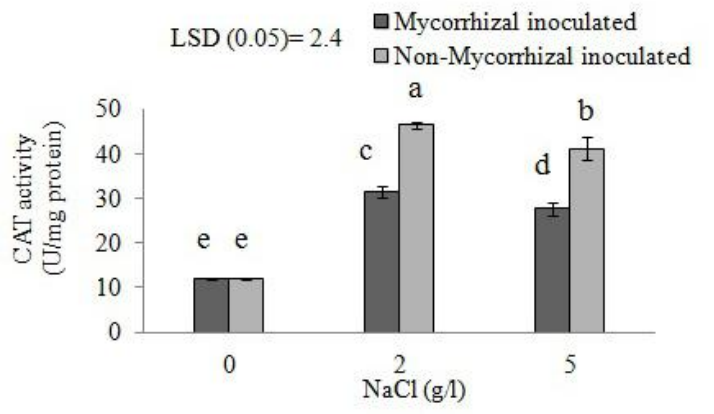

Figure 4; CAT activity in Stevia rebaudiana Bertoni plants inoculated with AMF or non-AMF subjected to three different levels of $\mathrm{NaCl}$ induced salt stress. Mean pairs for each treatments followed by different letters are significantly different $(\mathrm{P}<0.05)$ by LSD test; $\mathrm{n}=3$.

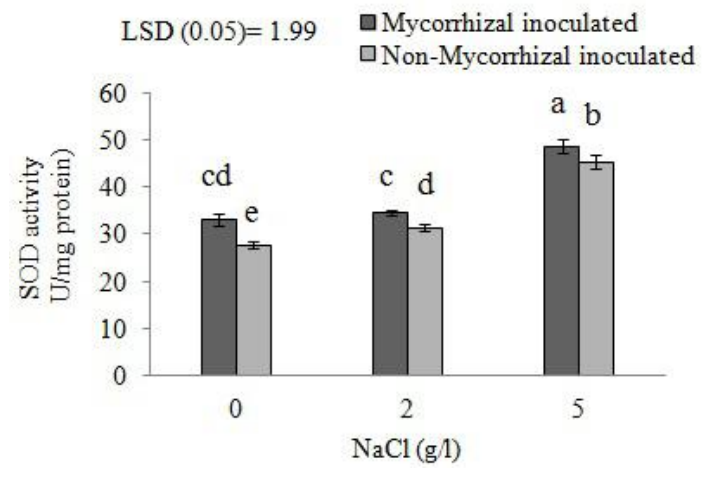

Figure 6; SOD activity in Stevia rebaudiana Bertoni plants inoculated with AMF or non-AMF subjected to three different levels of $\mathrm{NaCl}$ induced salt stress. Mean pairs for each treatments followed by different letters are significantly different $(\mathrm{P}<0.05)$ by LSD test; $\mathrm{n}=3$. 


\begin{tabular}{|c|c|c|c|c|c|c|c|c|}
\hline & 1 & 2 & 3 & 4 & 5 & 6 & 7 & 8 \\
\hline 1- Total chlorophyll content & 1 & $0.95^{* *}$ & $-0.91^{* *}$ & $-0.94^{* *}$ & $-0.94^{* *}$ & $-0.85^{* *}$ & $-0.95^{* *}$ & $-0.69^{* *}$ \\
\hline 2- Photosynthesis efficiency & & 1 & $-0.97^{* *}$ & $-0.97^{* *}$ & $-0.98^{* *}$ & $-0.83^{* *}$ & $-0.90^{* *}$ & $-0.67^{* *}$ \\
\hline 3- Electrolyte leakage (EL) & & & 1 & $0.95^{* *}$ & $0.97^{* *}$ & $0.74^{* *}$ & $0.81^{* *}$ & $0.74^{* *}$ \\
\hline 4- $\mathrm{H}_{2} \mathrm{O}_{2}$ & & & & 1 & $0.96^{* *}$ & $0.78^{* *}$ & $0.84^{* *}$ & $0.62^{* *}$ \\
\hline 5-MDA & & & & & 1 & $0.85^{* *}$ & $0.89^{* *}$ & $0.67^{* *}$ \\
\hline 6-CAT & & & & & & 1 & $0.95^{* *}$ & $0.34^{\mathrm{ns}}$ \\
\hline 7- POX & & & & & & & 1 & $0.55^{*}$ \\
\hline 8- SOD & & & & & & & & 1 \\
\hline
\end{tabular}

**Correlation is significant at the 0.01 level, *Correlation is significant at the 0.05 level and ${ }^{\text {ns }}$ Correlation is non- significant

Table1. Correlation coefficients among measured traits of AMF and non-AMF inoculated stevia plants under salt stress

The AMF inoculation of plants has been reported to have a considerable role in tolerance against adverse environmental conditions such as salt stress ${ }^{[1,8,10,13,17,32]}$. Salinity stress as shown in the result has affected some physiological attributes like chlorophyll content, photosynthesis efficiency of AMF and non-AMF inoculated stevia plants in a negative way, but those negative effects were more apparent in non-AMF inoculated plants. However, these physiological attributes clearly proves that the inoculation with mycorrhiza Glomus intraradices has a significant positive improving effect on stevia plants under control and salinity stressed conditions. Higher chlorophyll content in leaves of mycorrhizal plants under saline conditions has also been reported by various authors ${ }^{[1,2,5,11,24,26,34]}$. Similarly, Kumar et al. (2013) reported that AMF inoculation improved some physiological attributes such as chlorophyll content of $\mathrm{Na}_{2} \mathrm{SO}_{4}$ salt stressed Jatropha over non-inoculated plants ${ }^{[17]}$. Decline in the ratio of Fv/Fm (Chlorophyll fluorescence) in the leaves of stevia caused by salinity stress could be mitigated by AMF symbiosis, which was in line with the reports of Sheng et al. (2008) in maize plants ${ }^{[26]}$, where the higher ratio of Fv/Fm was observed in the leaves of AMF treated plants than those of non-AMF inoculated plants. Improvement of chlorophyll activity by AMF inoculated stevia grown under salinity stress is in confirmation with the findings of Kadian et al. (2013) who reported that the improved chlorophyll content of Cyamopsis tetragonoloba (L.) inoculated by AMF may be due to increased activity of specific enzymes involved in its biosynthesis ${ }^{[15]}$. Our results of mitigation in electrolyte leakage (EL) of leaves due to inoculation of stevia plants with AMF under salinity stress are in confirmation with the findings of Kaya et al. (2009) where the enhanced EL in leaves of Capsicum annum as a result of $\mathrm{NaCl}$ induced salinity stress was ameliorated in AMF-inoculated plants ${ }^{[16]}$. According to our results, AMF inoculated plants exhibited salinity tolerance by showing reduced EL over non-AMF plants at all salinity levels. Salinity stress was able to affect key physiological and biochemical processes of stevia, as indicated by measurements of some indices, e.g., lipid peroxidation (MDA production), electrolyte leakage, photosynthesis and different antioxidant enzymes (SOD, POX and CAT).

Electrolyte leakage (EL) showed very significant negative correlation with photosynthesis efficiency $\left(-0.98^{* *}\right)$ and very significant positive correlation with MDA production $\left(0.97^{* *}\right)$ (Table 1) suggesting that, the effect of salinity was most deleterious in accordance with the results for Fv/Fm, EL and MDA production (lipid peroxidation). The increased EL showed a positive correlation with $\mathrm{H}_{2} \mathrm{O}_{2}$ and MDA accumulation, suggesting that the detrimental effects of salt stress were associated with oxidative stress. In this study, AMF-inoculated stevia exhibited lower $\mathrm{H}_{2} \mathrm{O}_{2}$ and $\mathrm{MDA}$ content than non-AMF -inoculated plants under salt stress, supporting the positive role of AMF fungi in ameliorating of $\mathrm{NaCl}$ induced salt stress. Higher efficiency of photosynthesis (Fv/Fm) in AMF - inoculated stevia plants contribute to $\mathrm{NaCl}$ induced salt stress tolerance through lowering the components of oxidative stress $\left(\mathrm{H}_{2} \mathrm{O}_{2}\right.$ and MDA contents). Lower leakage values, $\mathrm{H}_{2} \mathrm{O}_{2}$ and MDA contents in AMF- inoculated stevia also supports the role of AMF fungi in enhancing the salt tolerance in stevia by activating the production of some antioxidant enzymes. Similar observations of the reduced of $\mathrm{H}_{2} \mathrm{O}_{2}$ and MDA contents caused by AMF inoculation which is possibly because of the increased activity 
of antioxidants in AMF-inoculated plants grown under salinity stress, have earlier been reported by Hashem et al. (2015) in Panicum turgidum Forssk. Our results of salinity induced enhanced in the activities of SOD and POX in stevia corroborate with the findings of Hashem et al. (2015) for Panicum turgidum Forssk and Alqarawi et al. (2014) for Ephedra aphylla Forssk ${ }^{[1,13]}$, however, further increase of these enzymes in AMF inoculated plants was observed only in terms of SOD activity, in the present study. Results of an increase in leaf CAT activity under low salinity followed by a decrease under high salinity in non-AMF and AMF inoculated stevia plants in our study support the findings of Yang et al. (2014) ${ }^{[32]}$, however, in contrast to their results, mycorrhizal plants demonstrated lower activities of CAT and POX than non-mycorrhizal plants, meaning that AMF fungi could not induce more activities of these enzymes in stevia under salt stress. The results of alleviation of salinity-induced detrimental effects through arbuscular mycorrhizal fungus in this study come in compliance with those of other researchers in Ephedra aphylla Forssk (Alqarawi et al. 2014) and Panicum turgidum Forssk (Hashem et al. 2015) $)^{[1,13]}$.

\section{Conclusion}

$\mathrm{NaCl}$ stress resulted in the collapse of Stevia growth due to its evident impacts on the physiological and biochemical indices studied. Salt stress, also increased production of hydrogen peroxide and lipid peroxidation causing damage to the cell membrane. These results evidence that, AMF mitigated the salt stress induced alterations by its positive effect on photosynthesis and antioxidant defense system, thereby enhancing plant efficiency and protecting cells from oxidative damage. Our study demonstrated that to reduce the unfavorable effects of salinity on stevia plants, the use of AM technology could be considered as a biological method for improving stevia production under salinity stress.

\section{Acknowledgments}

We wish to thank Trends in Horticulture editorial office for giving us the opportunity to submit this research article. We also thank all people who helped us to complete this study and wish to thank the college of agriculture, Isfahan University of Technology and Department of Agriculture, Iranian Research Organization for Science and Technology, (IROST) for allowing to conduct this study.

\section{References}

1. Alqarawi AA, Abd Allah EF, Hashem A (2014) Alleviation of salt-induced adverse impact via mycorrhizal fungi in Ephedra aphylla Forssk. Journal of Plant Interactions. 9(1): 802- 810.

2. Arya A, Buch H (2013) Response of arbuscular mycorrhizal fungi on growth and chlorophyll content of three varieties of Gossypium herbaceum L. Plant Pathol Quar. 3: 54-57.

3. Bhosale KS, Shinde BP (2011) Influence of arbuscular mycorrhizal fungi on proline and chlorophyll content in Zingiber officinale Rosc grown under water stress. Indian J Fundam Appl Life Sci. 1: 172-176.

4. Cantabella D, Piqueras A, Acosta-Motos JR, Bernal-Vicente A, Hernandez JA, Diaz-Vivancos P (2017) Salt tolerance mechanisms induced in Stevia rebaudiana Bertoni: Effects on mineral nutrition, antioxidative metabolism and steviol glycoside content. Plant Physiology and Biochemistry. Doi: 10.1016/j.plaphy.2017.04.023

5. Colla G, Rouphael Y, Cardarelli M, Tullio M, Rivera CM, Rea E (2008) Alleviation of salt stress by arbuscular mycorrhizal in zucchini plants grown at low and high phosphorus concentration. Biology and Fertility of Soils. 44: 501-509.

6. Cony MA, Trione SO (1998) Inter and intraspecific variability in Prosopis flexuosa and P. chilensis seed germination under salt and moisture stress. J. Arid Environ. 40: 307-317

7. Creus CM, Sueldo RJ, Barassi CA (1998) Water relations in Azospirillum inoculated wheat seedlings under osmotic stress. Can J Bot. 76: 238-244.

8. Elhindi KM, El-Din AS, Elgorban AM (2017) The impact of arbuscular mycorrhizal fungi in mitigating salt-induced adverse effects in sweet basil (Ocimum basilicum L.). Saudi Journal of Biological Sciences. 24: 170-179.

9. Estrada B, Aroca R, Barea JM, Ruiz-Lozano JM (2013) Native arbuscular mycorrhizal fungi isolated from a saline habitat improved maize antioxidant systems and plant tolerance to salinity. Plant Sci 201: 42-51.

10. Evelin H, Kapoor R, Giri B (2009) Arbuscular mycorrhizal fungi in alleviation of salt stress: a review. Annals of Botany. 104: 1263- 1280 .

11. Giri B, Mukerji KG (2004) Mycorrhizal inoculant alleviates salt stress in Sesbania aegyptiaca and Sesbania 
grandiflora under field conditions: evidence for reduced sodium and improved magnesium uptake. Mycorrhiza 14: 307-312.

12. Hajar EWI, Sulaiman AZB, Sakinah AMM (2014) Assessment of heavy metals tolerance in leaves, stems and flowers of Stevia rebaudiana plant. Procedia Environ. Sci. 20: 386-393.

13. Hashem A, Abd-Allah EF, Alqarawi AA, Aldubise A, Egamberdieva D (2015) Arbuscular mycorrhizal fungi enhances salinity tolerance of Panicum turgidum Forssk by altering photosynthetic and antioxidant pathways. Journal of Plant Interactions 10(1): 230-242.

14. Ityel E, Lazarovitch N, Silberbush M, Ben-Gal A (2012) An artificial barrier to improve root-zone conditions for horticultural crops: response of pepper plants to matric head and irrigation water salinity. Agric. Water Manage. 105: 13-20

15. Kadian N, Yadav K, Badda N, Aggarwal A (2013) Application of arbuscular mycorrhizal fungi in improving growth and nutrient of Cyamopsis tetragonoloba (L.) Taub. Under saline soil. Int J Agron Plant Prod. 4: 2796-2805.

16. Kaya C, Ashraf M, Sonmez O, Aydemir S, Tuna AL, Cullu MA (2009) The influence of arbuscular mycorrhizal colonization on key growth parameters and fruit yield of pepper plants grown at high salinity. Scientia Horticulturae. 121: 1-6.

17. Kumar A, Sharma S, Mishra S, Dames JF (2013) Arbuscular mycorrhizal inoculation improves growth and antioxidative response of Jatropha curcas (L.) under Na2SO4 salt stress. Plant Biosystems- DOI: $10.1080 / 11263504.2013 .845268$

18. Ozden M, Demirel U, Kahraman A (2009) Effects of proline on antioxidant system in leaves of grapevine (Vitis vinifera L.) exposed to oxidative stress by H2O2. Scientia Horticulturae. 119: 163- 168.

19. Padmaja M, Sravanthi M, Hemalatha KPJ (2011) Evaluation of antioxidant activity of two Indian medicinal plants. J Phytol 3(3): 86-91.

20. Pol J, Ostra EV, Karasek P, Roth M, Benesova K, Kotlaríkova P, Caslavsky J (2008) Comparison of two different solvents employed for pressurised fluid extraction of stevioside from Stevia rebaudiana: methanol versus water. Anal. Bioanal. Chem. 388: 1847-1857.

21. Ramesh K, Virendra S, Megeji NW (2006) Cultivation of stevia [Stevia rebaudiana (Bert.) Bertoni]: a comprehensive review. Adv. Agron. 89: 137-177

22. Reis M, Coelho L, Santos G, Kienle U, Beltrao J (2015) Yield response of stevia (Stevia rebaudiana Bertoni) to the salinity of irrigation water. Agricultural Water Management 152: 217- 221.

23. Ruiz-Lozano JM, Porcel R, Azcon C, Aroca R (2012) Regulation by arbuscular mycorrhizae of the integrated physiological response to salinity in plants: new challenges in physiological and molecular studies. J Exp Bot 63 : 4033-4044.

24. Sannazzaro AI, Ruiz OA, Albetro EO, Menedez AB (2006) Alleviation of salt stress in Lotus glaber by Glomus intraradies.Plant and Soil 285: 279-287.

25. Shannon MC, Grieve CM (1999) Tolerance of vegetable crops to salinity. Scientia. Horticulturea. 78: 5-38

26. Sheng M, Tang M, Chan H, Yang B, Zhang F, Huang Y (2008) Influence of arbuscular mycorrhizae on photosynthesis and water status of maize plants under salt stress. Mycorrhiza. 18: 287-296.

27. Sherstha YH, Ishii T, Kadoya K (1995) Effect of vesicular-arbuscular mycorrhizal fungi on the growth, photosynthesis, transpiration and the distribution of photosynthates of bearing Satsuma mandarin trees. J. Jpn. Soc. Hortic. Sci. 64: 517-525.

28. Tanwar BS (2003) Saline Water Management for Irrigation. International Commission on Irrigation and Drainage, New Delhi, India.

29. Velikova V, Loreto F (2005) On the relationship between isoprene emission and thermo tolerance in Phragmites ausrralis leaves exposed to high temperatures and during the recovery from a heat stress. Plant, Cell and Environment. 28: 318-327.

30. Wang F, Zeng B, Sun Z, Zhu C (2009) Relationship between proline and $\mathrm{Hg}+2$ - induced oxidative stress in tolerant rice mutant. Archives of Environmental Contamination and Toxicology 56: 723-731.

31. Wu N, Li Z, Wu F, Tang M (2016) Comparative photochemistry activity and antioxidant responses in male and female Populus cathayana cutting inoculated with arbuscular mycorrhizal fungi under salt. Scientific Reports 6 , 37663. DOI: 10.1038/srep37663

32. Yang SJ, Zhang ZL, Xue YX, Zhang ZF, Shi SY (2014) arbuscular mycorrhizal fungi increase salt tolerance of apple seedlings. Botanical Studies 55:70. http://www.as-botanicalstudies.com/content/55/1/70

33. Zeng J, Chen A, Li D, Yi B, Wu W (2013) Effect of salt stress on the growth, physiological responses, and glycoside contents of Stevia rebaudiana Bertoni. J. Agric. Food Chem. dx.doi.org/10.1021/jf401237x.

34. Zuccarini P (2007) Mycorrhizal infection ameliorates chlorophyll content and nutrient uptake of lettuce exposed to saline irrigation. Plant, Soil and Environment 53: 283-289. 